\section{Beetling: a method for capturing trapdoor spiders (Idiopidae) using tethered beetles}

\author{
Victoria R. Smith \\ Cor J. Vink* \\ Robert H. Cruickshank \\ Adrian M. Paterson \\ Department of Ecology, \\ PO Box 85084, \\ Lincoln University, \\ Lincoln 7647, New Zealand \\ email: Vikki.Smith@lincolnuni.ac.nz \\ *and Canterbury Museum, \\ Rolleston Avenue, \\ Christchurch 8013, New Zealand
}

\section{Summary}

Collecting trapdoor spiders (Idiopidae) for research is difficult due to their deep, convoluted burrows and almost entirely fossorial life history. Digging idiopids out of their burrows is laborious, disturbs the environment, and can only be undertaken in open areas with soft soil. Here we describe "beetling": a quicker, easier method of capturing idiopids, using tethered beetles to lure the spiders from their burrows. Beetling was used to capture 123 individual Cantuaria spp. (Idiopidae) out of a total of 130 successfully throughout New Zealand during March-June, September, November, and December. We conclude that beetling is an effective method for the live capture of idiopids, despite some limitations such as the need to work at night, and to culture live beetles, but they are outweighed by the advantages of having a reliable, efficient method of capturing live spiders. Beetling could also be used to catch other fossorial invertebrates, such as lycosids and carabid larvae.

\section{Introduction}

Trapdoor spiders (Mygalomorphae: Idiopidae) are widespread in the southern hemisphere (Platnick 2014), and occupy almost entirely a fossorial niche. Idiopids dig underground burrows up to $49 \mathrm{~cm}$ deep (Irish 2001) from which they may never fully emerge. Their high site fidelity and low dispersal ability make Idiopidae particularly interesting from an ecological and evolutionary perspective (Bond \& Stockman 2008; Cooper et al. 2011), although their nocturnal, burrow-dwelling, life histories make collecting samples difficult.

Commonly used methods of collecting idiopids include digging (Irish 2001; Marples \& Marples 1972), and pitfall-trapping for males (Engelbrecht 2013) and nematode-infected females (Poinar \& Early 1990). While often successful, these methods have obvious drawbacks. Idiopid burrows are usually deep, often in hard soil, and become convoluted around rocks and tree roots, making digging laborious and difficult, as well as damaging to the burrow and surrounding area. Digging can also result in the accidental dismembering of the specimen. Pitfall trapping male Idiopidae can be successful when they are searching for females at specific times during the year. For example, male Cantuaria Hogg, 1902 often leave their burrows in autumn and winter to look for females (Irish 2001), whereas female idiopids typically never leave their burrows (Engelbrecht 2013). Thus pitfall trapping is highly seasonal and is only suitable for the collection of male Idiopidae. The males collected using pitfall traps may be immigrants and therefore pitfall trapping a male does not enable reliable determination of the source population, as males may wander far from their source population in search of females. Both pitfall trapping and digging require carrying bulky and sometimes heavy equipment (shovels or pitfall traps), and trapping often requires multiple visits to a location at least a week apart.

Methods of trapping predatory vertebrates sometimes involve using live bait; for example, birds of prey may be trapped using a live prey species, such as a mouse or bird, inside a bal-chatri trap (a cage covered with nooses) (Berger \& Mueller 1959; Dykstra et al. 2012). Food bait is often used to attract invertebrates for collection (for example, baiting pitfall traps with squid (Seldon \& Beggs 2010), but few published studies have used live bait. Burrowing arachnids have been trapped using a specially designed container, the efficacy of which may be increased by adding a live insect bait inside a vial (Henschel 1991), however, the success of this method relies on the individual leaving its burrow to encounter the trap.

We describe a new method for capturing trapdoor spiders called "beetling": the use of tethered mealworm beetles Tenebrio molitor Linnaeus, 1758 to attract female trapdoor spiders, in this case of the genus Cantuaria, to exit their burrows for easy collection. Cantuaria is a particularly speciose genus ( $>42$ species) that is endemic to New Zealand, and exhibits typical idiopid habitat selection, preferring clay banks or damp forests. Our method is simple to execute, requires minimal equipment, and is successful when used to capture Cantuaria spp. While digging out a trapdoor spider usually takes about 20 minutes (pers. obs; M. Wakelin pers. comm.), and pitfall traps must be left out for at least a few days, a spider may be attracted instantly using a tethered beetle. Beetling was successfully used over three months to collect Cantuaria spp. from populations around New Zealand.

\section{Methods}

Beetles

Tenebrio molitor beetles were obtained from an existing culture in the Department of Ecology at Lincoln University, New Zealand. Mealworms (T. molitor larvae) are easily obtained from pet shops and animal feed suppliers in many countries. The beetles were maintained on a porridge oat substrate, with occasional pieces of bread, carrot, broccoli, dry meat, or dog biscuit added to the substrate for extra nutrition. Beetles, larvae, and pupae were kept separate to avoid cannibalism.

Before use in the field, a single beetle was selected based on size (smaller beetles for smaller species of Cantuaria) and tethered by passing one loop of cotton thread around the joint between the thorax and abdomen and behind the first pair of legs, if the beetle was needed immediately and was handled particularly carefully (Fig. 1A). A single loop was also used with other invertebrates, such as amphipods, that do not have narrow joints between body segments (Fig. 1B). 


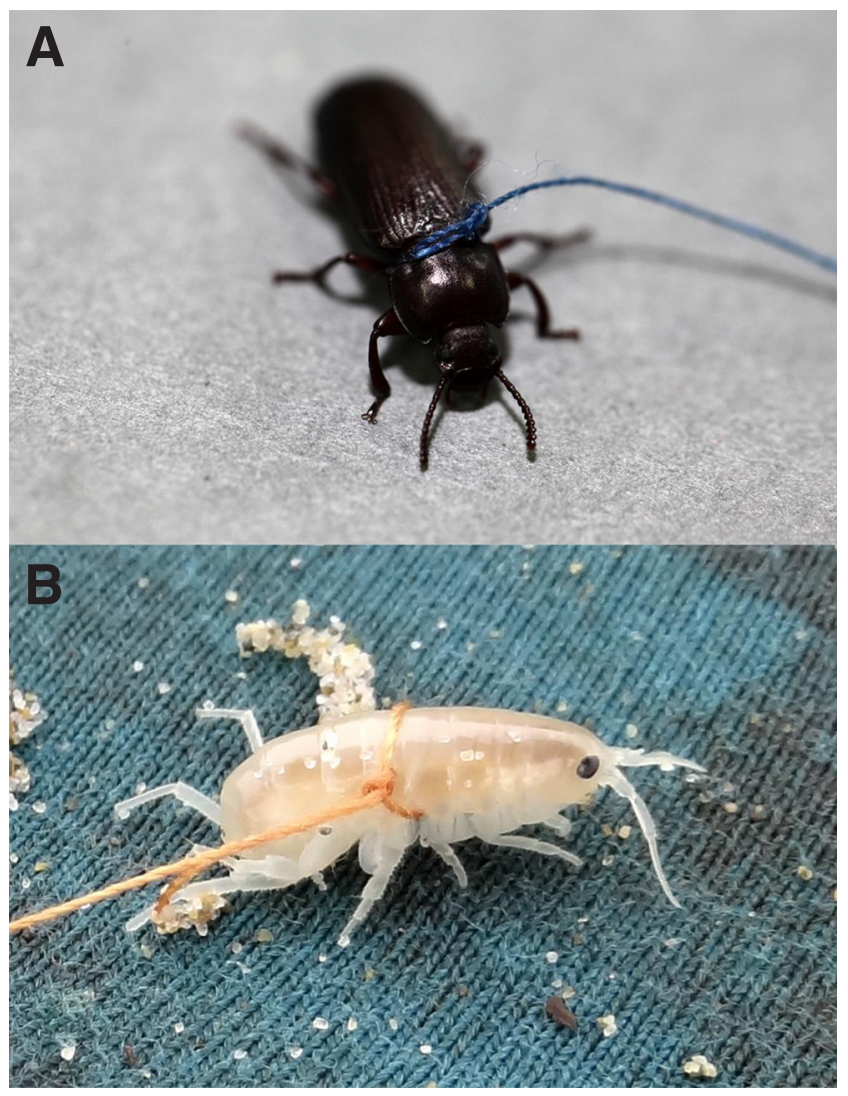

Fig. 1: A Tenebrio molitor beetle, and $\mathbf{B}$ talitrid amphipod, tethered with one loop of cotton thread.

Alternatively, one loop between the head and thorax, and another between the thorax and abdomen, could be used (Fig. 2). Using two loops of thread rather than one enables greater control over the beetle, is less likely to cause damage, and prevents the beetle from escaping. The easiest way to get the thread around the beetle is to hold it by the abdomen and pass a loop of thread, tied with a single overhand knot, over the beetle's head. The knot can be tightened behind the pronotum, then the thread is passed behind the front legs (which the beetle will lift if the abdomen is tilted back), crossed over, and tied with two overhand knots behind the thorax (Fig. 2). The beetle can be tethered to a post or stick in the ground until needed, but care must be taken not to place it near spider burrows. After the same beetle was used for three consecutive nights, its tether was removed and it was returned to the source culture.

\section{Identifying burrows}

Cantuaria were sampled from Southland, West Coast, Otago, Canterbury, Nelson, Tasman, Wellington, and Manawatu-Whanganui regions in New Zealand (Fig. 4). Populations were located using local knowledge, and records from Forster \& Wilton (1968) and Irish (2001). Burrows were identified and marked with lollipop sticks painted with red Dulux Spraypak ${ }^{\mathrm{TM}}$ Dazzle fluorescent paint. We aimed to locate and mark at least seven burrows per population; from these seven burrows, at least three specimens could usually be caught.
Attracting a spider

The beetle was placed adjacent to the lid or mouth of a selected spider burrow, with the thread kept loose to enable movement. If the beetle strayed too far from the lid, or appeared to be about to walk onto the lid itself, a quick tug on the thread would pull the beetle away from the substrate. If the thread was tugged too slowly or held taut, the beetle would grip onto the substrate, which could result in too much disturbance, and discourage the spider from leaving its burrow.

\section{Collecting}

If beetling was successful, the spider would leave the burrow to strike. At this point, the handler would remove the beetle and immediately drive a trowel into the ground between the spider and its burrow to block the entrance. Care was taken to point the trowel down the burrow, rather than across it, to avoid mutilating the specimen. If the trowel was inserted quickly enough, the spider would stop moving.

\section{Results and Discussion}

\section{Collecting success}

Between December 2013 and November 2014, 130 Cantuaria specimens were collected from 102 populations located throughout the South Island and lower North Island of New Zealand (see Fig. 4). Beetling was conducted as far south as Stewart Island, and as far north as Makirikiri (near Whanganui) (see Fig. 4). Beetling was only attempted in the months of December, March, April, May, June, September, and November. Efforts were not evenly distributed throughout these months; in March, April, May, and June, collection was attempted approximately every night,

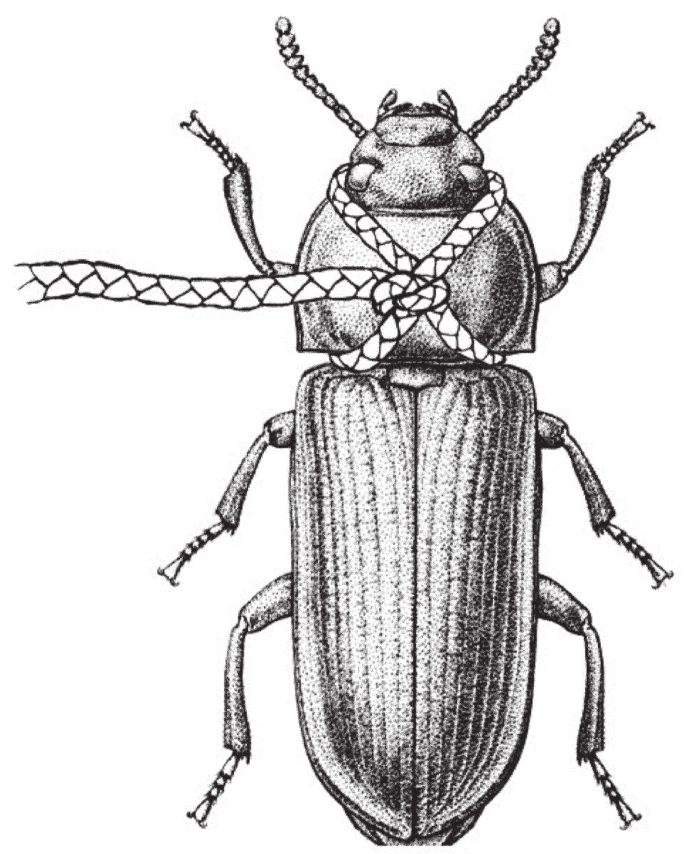

Fig. 2: A beetle wearing a two-looped harness. 


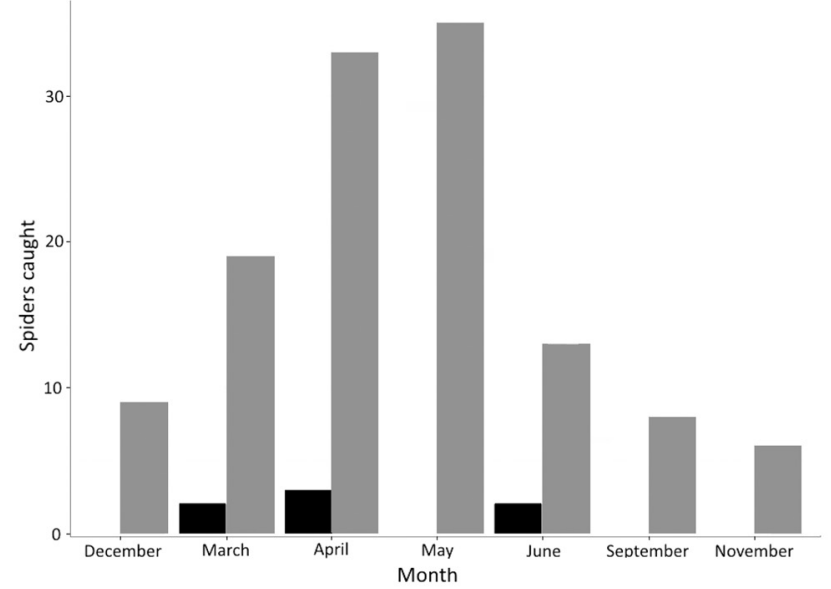

Fig. 3: The total number of spiders caught per month, at all localities, when beetling was attempted. Grey bars show the number of spiders caught using beetling, and black bars show the number of spiders caught using another method after beetling had failed. Collecting effort was unevenly distributed between months. Graph constructed in R (R Core Team 2013) using ggplot2 (Wickham 2009).

whereas in December, September, and November, only the occasional collection was attempted. Beetling was always attempted first, and was used successfully to collect 123 individuals. If beetling proved unsuccessful in a population, digging would be attempted. In four populations, beetling failed to retrieve a spider, so individuals $(n=7)$ had to be collected by digging or using a carabid beetle. On two occasions, beetling worked for some individuals but not others, and on six occasions no specimens were retrieved; the spiders were not attracted to the beetle, and the ground was too hard for digging.

Beetling also lured other fossorial invertebrates from their holes, including tiger beetle larvae (Carabidae), wolf spiders (Lycosidae), other Mygalomorphae (Hexathelidae, Nemesiidae) and vagrant spiders (Zoropsidae).

\section{Beetle positioning}

The following observations are offered as a guide to beetle positioning: 1) Trapdoor burrows are surrounded by radial strands of silk (Irish 2001). If the beetle stands on one or more adjacent radial strands then the spider may strike immediately. 2) Allowing the beetle to walk once over the lid of the burrow appears to alert an otherwise unresponsive spider. 3) Pulling hard on the leash will cause the beetle to rear up, grasping at the substrate with its tarsi and causing more disturbance than leaving the leash slack and allowing the beetle to roam more freely. Varying the tautness of the leash (and therefore the amount that the beetle is pulling on the substrate) may entice an otherwise unresponsive spider. 4) The most successful beetle position appears to be with its head approximately $2 \mathrm{~mm}$ from the front of the lid, and the leash tight so that the beetle is attempting to claw its way towards the entrance, but is not gaining any headway. 5) If the beetle must be repositioned, snapping it quickly up prevents it from dislodging a large amount of substrate and disturbing the spider.
Waiting time

Occasionally, spiders were found with their tarsi outside the entrance to the burrow. Any movement around the burrow by the beetle handler would result in the spider withdrawing its tarsi. However, if the beetle could be lowered to the ground next to a tarsus, the spider would usually strike within approximately ten seconds. Other times between beetle deployment and spider strike were highly variable, but usually a spider would strike within ten minutes. Spiders took longer to strike if the beetle moved more slowly, or if the researcher's movements disturbed the spider.

Some Cantuaria reacted to the beetle defensively, by pulling their trapdoor lids inwards. Defensive Cantuaria were easily caught by thrusting the trowel into the ground just as the trapdoor was pulled in, while the spider was still near the lid.

\section{Alternative methods of collecting trapdoor spiders}

Repeatedly dragging a piece of grass past the burrow's entrance, as commonly used to lure burrowing spiders, such as theraphosids (Hamilton 2008), often caused the spider to half-leave the burrow and strike, although the spider would not leave the burrow for long enough to facilitate capture. Carabid beetles Megadromus antarcticus Chaudoir, 1865 and M. guerinii Chaudoir, 1865 were also harnessed and deployed down burrows to chase the inhabitant out; carabids are highly aggressive, and often larger than the spiders. Tethered carabids were excellent at causing Stanwellia kaituna Forster, 1968 to leave their burrows, but success was limited with Cantuaria spp., and often resulted in an injury to the spider. On one occasion, the carabid used its jaws to pull a female from its burrow. When deployed down a neighbouring burrow, however, the same carabid was eaten by a mother spider, possibly in defence of her spiderlings.

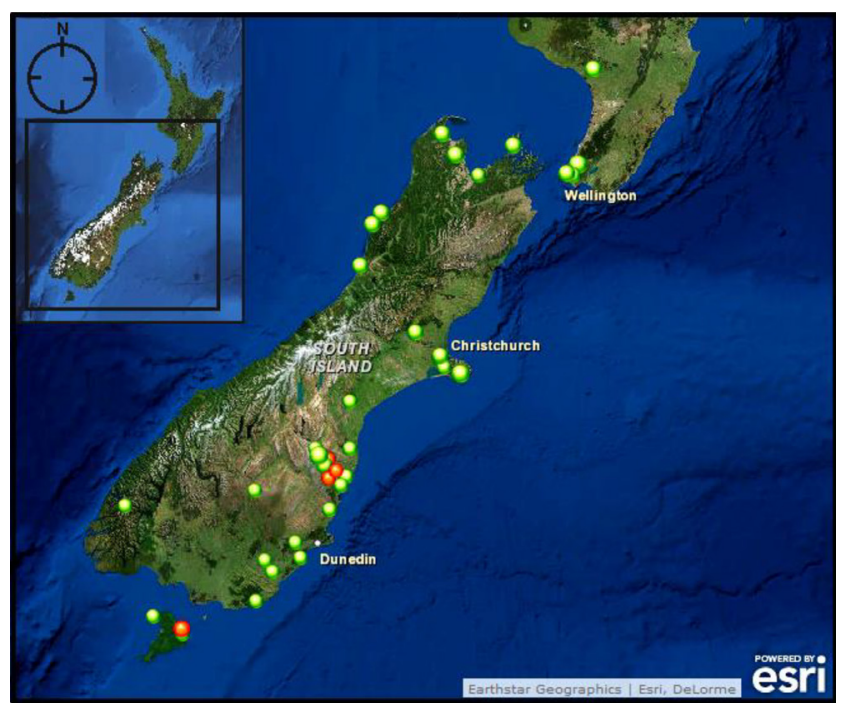

Fig. 4: Map of collecting sites where beetling was successful (green/light grey markers) and unsuccessful (red/dark grey markers). 


\section{Limitations of beetling}

On cold nights, T. molitor would often move too slowly to be attractive to Cantuaria. Warming the beetle with the researcher's body heat would temporarily encourage faster movement. Occasionally, beetles would stop and eat or drink from the substrate rather than moving; stationary beetles did not attract Cantuaria. Tethering the beetle on the ground enables it to take moisture and minerals from the soil prior to commencing beetling, reducing its tendency to stop moving.

Driving the trowel into the ground as the spider emerges from its burrow can sometimes result in mutilation of the specimen, particularly if it is large. Care must be taken to drive the trowel in the same direction as the burrow, rather than across it, to avoid damaging the specimen. In particularly hard ground, the trowel may not cut through the soil quickly enough to prevent the spider from escaping. However, hard soil also increases the difficulty of digging, whether for pitfall trap placement, or for directly removing a spider from its burrow.

Tenebrio molitor are easy to obtain, simple to culture (Martin, Rivers \& Cowgill 1976), and larvae are resilient to field conditions, such as temperature fluctuation (pers. obs). However, T. molitor do require space, maintenance, and must be handled carefully to prevent damage. Recently pupated, teneral beetles are particularly fragile due to their soft cuticle, and therefore should not be tethered. Laboratory-raised T. molitor may also be prohibited from some protected offshore islands (e.g. Codfish Island), so other invertebrate bait would be required to capture idiopids (e.g. amphipods, Fig. 1B).

Unlike current popular methods for collecting trapdoor spiders, such as digging, beetling must be conducted at night, when the spiders are most active. Several populations can be located during the day, but collection of individuals is limited to two to three populations only. Spider responsiveness appeared to decline after a few hours of darkness.

The limitations of using tethered beetles to collect idiopid specimens are minor in comparison to the advantages. Beetling is a simple and easily executed method to lure both male and female idiopids from their burrows without disturbing the burrows or surrounding habitat.

\section{Wider implications}

In addition to luring Cantuaria from their burrows, beetling also attracted other species of fossorial predatory invertebrate. We did not investigate beetling as a possible method of capturing other invertebrates, but it could potentially be used to capture any fossorial predatory animal that relies on tactile information from the surface to detect prey.

\section{Acknowledgements}

We are grateful to Ashley White, Kevin McGinn, Jamie Cooper, and Tasha Shelby for their assistance in the field. Many thanks to Myles MacKintosh for all his help. Alex Wooton created the artwork for Fig. 2. The Department of Conservation supplied permits and assistance, particularly in Stewart and Codfish Islands, and Phred Dobbins in particular was very helpful in assisting us with protocols, transport and quarantine.

\section{References}

BERGER, D. D. \& MUELLER, H. C. 1959: The bal-chatri: a trap for the birds of prey. Bird-Banding 30: 18-26.

BOND, J. E. \& STOCKMAN, A. K. 2008: An integrative method for delimiting cohesion species: finding the population-species interface in a group of Californian trapdoor spiders with extreme genetic divergence and geographic structuring. Systematic Biology 57: 628-646.

COOPER, S. J. B., HARVEY, M. S., SAINT, K. M. \& MAIN, B. Y. 2011: Deep phylogeographic structuring of populations of the trapdoor spider Moggridgea tingle (Migidae) from southwestern Australia: evidence for long-term refugia within refugia. Molecular Ecology 20: 3219-3236.

DYKSTRA, C. R., MAYS, H. L., HAYS, J. L., SIMON, M. M. \& WEGMAN, A. R. 2012: Sexing adult and nestling red-shouldered hawks using morphometrics and molecular techniques. Journal of Raptor Research 46: 357-364.

ENGELBRECHT, I. 2013: Pitfall trapping for surveying trapdoor spiders: the importance of timing, conditions and effort. Journal of Arachnology 41: 133-142.

FORSTER, R. R. \& WILTON, C. L. 1968: The spiders of New Zealand. Part 2: Ctenizidae, Dipluridae, Migidae. Otago Museum Bulletin 2 : $1-180$.

HAMILTON, D. E. 2008: Combining direct methods (PIT tags and radiotelemetry) with an indirect method (mtDNA) to measure movement and dispersal at different scales in North American tarantulas (Aphonopelma spp.). Ph.D. thesis, Texas Tech University.

HENSCHEL, J. R. 1991: A trap to capture burrowing arachnids. Journal of Arachnology 19: 150-152.

IRISH, L. L. 2001: The minefield spiders. Dunedin: Biodiversity Solutions.

MARPLES, B. J. \& MARPLES, M. J. 1972: Observations on Cantuaria toddi and other trapdoor spiders (Aranea: Mygalomorpha) in Central Otago, New Zealand. Journal of the Royal Society of New Zealand 2: 179-185.

MARTIN, R. D., RIVERS, J. P. W. \& COWGILL, U. M. 1976: Culturing mealworms as food for animals in captivity. International Zoo Yearbook 16: 63-70.

PLATNICK, N. I. 2014: The world spider catalog, version 15.0. New York: American Museum of Natural History, online at 10.5531/db.iz.0001

R CORE TEAM 2013: $R$ : A language and environment for statistical computing, version 3.0.2. Vienna: R Foundation for Statistical Computing, online at http://www.R-project.org

POINAR, Jr., G. O. \& EARLY, J. W. 1990: Aranimermis giganteus n. sp. (Mermithidae: Nematoda), a parasite of New Zealand mygalomorph spiders (Araneae: Arachnida). Revue de Nematologie 13: 403-410.

SELDON, D. S. \& BEGGS, J. R. 2010: The efficacy of baited and live capture pitfall traps in collecting large-bodied forest carabids. New Zealand Entomologist 33: 30-37.

WICKHAM, H. 2009: ggplot2: Elegant graphics for data analysis (use $R$ !). New York: Springer. 